\title{
Remote Sound Data Collection and Analysis Based on LabVIEW
}

\author{
Hongke Xu, Pei Wang, Chuang Gao, Shan Lin,Jing Wang \\ School of Electronic and Control Engineering, Chang'an University, Xi'an, 710064, China \\ e-mail: xuhongke@chd.edu.cn, wangpei24@gmail.com.
}

\begin{abstract}
The detection and processing of the sound signal are significant to the fault diagnosis in industrial processing and production quality monitoring. The current sound signal collection system is mainly used to collect signal. It can't meet the demand of the engineers on special occasions to master the process of the signal acquisition from remote timely. In order to solve this problem, this article designed a real-time monitoring system which can collect the signal by a sound card and implement the analysis of the sound signal's power spectrum and phase spectrum, storage and playback. In addition, it can use the TCP/IP protocol to transmit the signal to remote engineers timely. Practice shows us that the system has the characters of simple structure, steady data transition, good reliability, low cost and friendly interaction.
\end{abstract}

Keywords-Virtual instruments, TCP/IP signal communication, Data acquisition, Remote monitor.

\section{INTRODUCTION}

To detect and process the sound signals exist extensively in the industrial production. The sound signals which have the characters of real-time, accuracy and stability, show its unique superiority compare to other signals. It can provide accurate basis of data for predictions of the industry field conditions. ${ }^{[1]}$ Thus, it is significant to the fault diagnosis in the industrial processing and production quality monitoring. On some danger occasions or poor conditions, it is hardly to send the sound signal timely by using some traditional devices to the remote engineers frequently, so that brings incalculable hidden trouble indirectly. In order to solve the problem, this paper designed a sound collection system which can be used to monitor remotely in real time. It is composed of two parts-the collection system and remote terminal. This paper studies and discusses the method of the system in hardware and software design and realization.

The design is a data acquisition system (DAQ) which is organized according to instruments design, including the data collection, processing and analysis. The signal processing and the constitution of the DAQ system is showing as the figure1 and figure2 separately.

The system processed the data which had been collected from the field. Subsequently, it showed the waveform on the font-panel and used the network to transit that provided the data to the engineers who can use the data to analyze and estimate remotely. The system implements the functions of data collection remotely, data display timely, analysis and storage. Thus, it provides a further research platform to forecast and diagnose faults in industrial field and monitor the product quality.

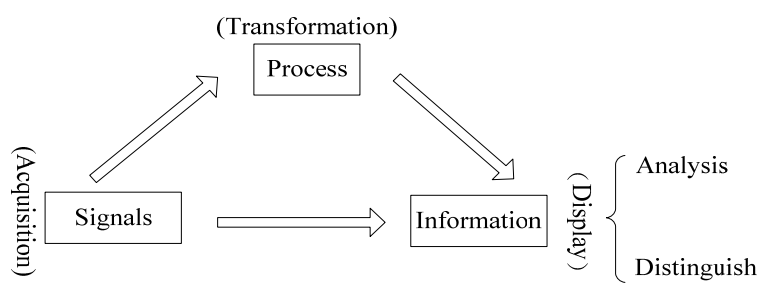

Figure1 The signal processing

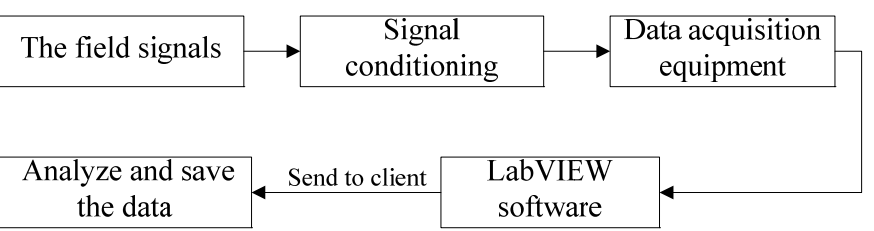

Figure 2 The system of a DAQ

\section{THE HARDWARE OF THE SYSTEM}

The system consists of five parts-a microphone, a PC sound card, a PC for field acquisition, LabVIEW application and a PC for remote monitoring. The structure of the system is as the figure3 shows here. The sound signal collects by a microphone can transform to electric signal through a sensor. Then using the sound card to do the AD transforms. After the digital processing, the data can be displayed, stored and analysed.

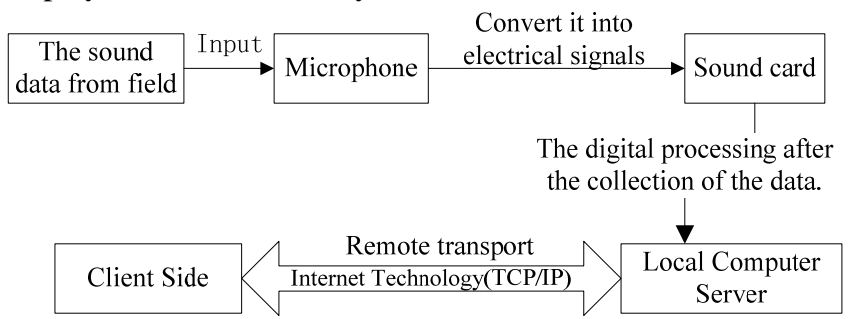

Figure3 The structure of the system

The sound card has the function of $\mathrm{AD}$ and $\mathrm{DA}$ conversion. It usually has two-channel and collects by 16 bits of precision and works in the audio range between 0.02 20 kHz. Meanwhile it meets requirements for the most engineering measurement and scientific experiments.

A sound card usually has a Line-In and Mic-In two input ports. The sound signal can be connected to the sound card by the two ports. 
However, the sound card input port Mic-In has stopping capacitors. The low frequency signal is attenuated severely especially for whose frequency is under $20 \mathrm{~Hz}$.So the input signal's frequency should be better ensure between $0.1 \sim 15 \mathrm{Kz}$. If we used the Line-In as the input port, it will be easier to introduce noise and overload because it has a preamp. Therefore, the design uses the Mic-In input port which has low input noise and good dynamic performance compared to the Line-In. In addition, the introduction of the measurement signal should use audio cable or shielded cable to reduce the influence of the noise. If the input signal level is higher than the sound card's design requirement it should be installed an attenuator between the sound card's input port and the measured signal that will be attenuated down to the input level no more than the sound card allowed.

\section{THE SOFTWARE OF THE SYSTEM}

\section{A. The overall design scheme of the system}

The total program of the system is divided into four major modules: data acquisition, data analysis and storage, data remote transmission and data broadcast. The data acquisition module is used to collect the real-time signal by using a sound card. The module of the data analysis and storage is to analyze the power spectrum and phase spectrum and to store the data separately. The function of the data remote transmission module is to send the real-time acquisition data to the client computer and can display the waveform conveniently on the it's font-panel. Lastly, the data broadcast module is to playback the sound data which has been stored in the computer disk.

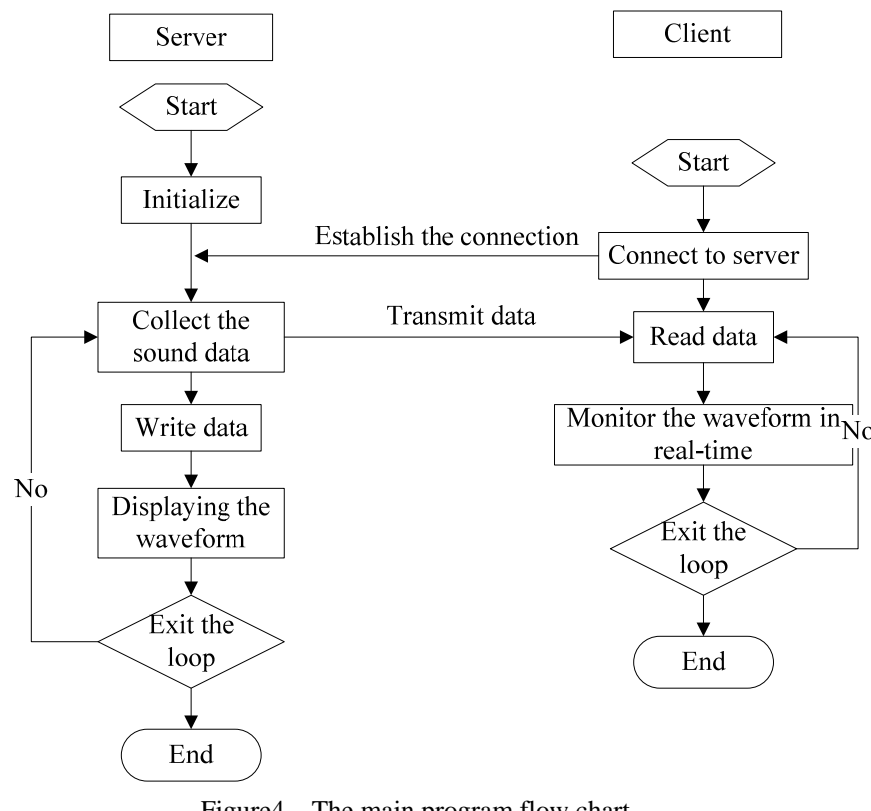

Figure4 The main program flow chart

\section{B. The interface design of the system}

According to the demand of the system interface design, the system used many controls like buttons, dialog boxes, waveforms, indicator lights and sliders. Therefore, it can provide the better Human Machine Interface. The interface of the system is as the figure 5 shows here. There are five push buttons on the top left corner. They are separately to control the collections and stops, playback and stop playback. The red one is to log out.

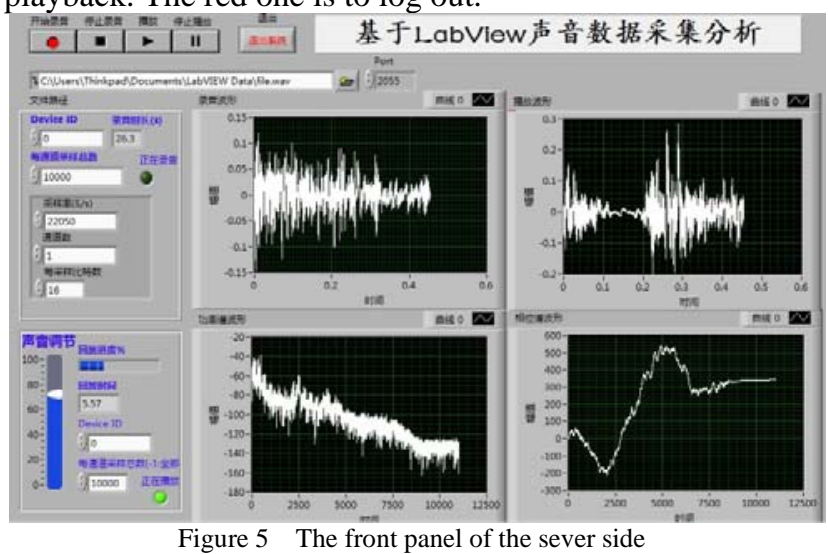

Under the control buttons, there is a path to save and broadcast the data. When the program is running for the first time, after push down the acquisition button at the server side, a dialog box appears prompting you to choose a path where to save the data. After the collection of data, it will save the acquisition data to the path where you chose automatically. The default file name and the format are "test" and ".wav" separately.

The input field under the title is port number of the sever. It must be assigned a port number for the sever and client side simultaneously when using the TCP note to communicate so that can assure establish communications correctly. The port can be assigned to a number or a string.Besides, the port number blow 1024 usually has a designated use. For instance: FTP, HTTP, SMTP and Telnet, etc. For this reason, it should better assign the number larger than 1024.

Blow the path setting, are the settings of the sampling attributes. It mainly includes the followings.

1) Sampler model: This attribute is assigned the model when collect a simple as a finite-sample or continuous sampling. The design chooses the continuous sampling so that the model can call the "sound input read.vi" repeatedly as required.

2) Sample frequency: There are three sample frequency: $11025 \mathrm{~S} / \mathrm{s}$ 、 $22050 \mathrm{~S} / \mathrm{s} 、 44100 \mathrm{~S} / \mathrm{s}$. The system accepts the default value- $22050 \mathrm{~S} / \mathrm{s}$.

3) Number of bits per sample: This attribute is resigned to the quality of every sample. It units in bits and the resolution is usually 16 bit or 8bit. The default value is 16bit. Besides, if the value of the sample frequency or the number of bits per sample is higher, the computer memory occupancy will be higher as well. 
4) Each channel sampling number: It is assigned to the sample number of each channel in the data buffer. The number should be large when we need sample continuously. So the design chooses $10000 / \mathrm{ch}$. Like the sample frequency the number should be an appropriate one if it is too large the computer memory occupancy will be too high.

5) Number of channels: The number of channels should accord with number which the sound card are supported. For most sound card, 1 is mono and 2 stereo. In order to calculate data and analyze spectrum the design uses the mono.

6) Device ID: It referred to the device's identity which we used in input or output. In most cases we chose the default value -0 . The option values are between 1 and $n-1$. The "n" referred to the number of the input or output devices on the computer. In addition, for the sake of showing the used time to engineers, there is an output box to show the collecting time.

Finally, in order to show the running status of the system clearly, two green indicator lights have been added to the panel. They are used to show the collection and playback status separately. Furthermore, there is a control of vertical slider that can adjust the playback's sound volume and another control-the horizontal slider can display the playback progress.

C. The program design of software system

The system can be divided by two independent parts- a server which is used to collect and send the sound signal and a client that can receive the data and display the sound signal's waveform. The program of the server part mainly contains three modules: the sound signal collection, transmission and playback module.

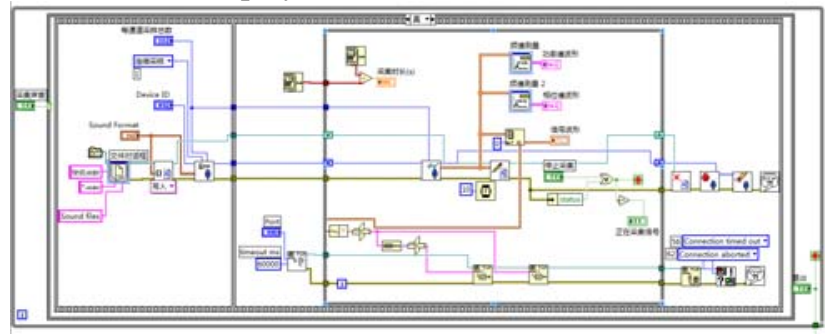

Figure6 The sound collection and transmission program

The program graph is as figure6 shows. All of the program is in a while loop, when you click the collect button, the property turns from true to false and goes into the condition loops. There is a Flat Sequence Structure in the condition loops. In the first frame of the structure are the configurations of the sound signal collection and the save of the data file. They are connected into the "Sound Input Configuration.vi" and the "Sound File Open.vi". The function of the "Sound Input Configuration.vi" is to configure the input devices, collect the sound data and send it to cache. The "Sound File Open.vi" is used to create a new file that can save the sound data. The second frame is to read the sound data which has been collected and to display it on the front panel then to save it as a ".wav" file.
It can send the data to the client by using the TCP node at last. On the other hand, it uses the "Sound Input Read.vi" to read the data and to display it on the front panel by the Digital Waveform Graph. Meanwhile, the "Spectrum Measurements.vi" can process the sound data that we can get the power spectrum and the phase spectrum of the sound signal. When the system finished the task of collection, it will call the "Sound File Close.vi" to close the file and stop the sound collection, clear all the sound data.

Eventually, it will write the elements of the sound data to the TCP node. Correspond to the client side, the server side uses two TCP data node to send data. One of them is used to send the length of the audio waveform array and the other one is to the data of the audio waveform array. In this way that can make sure to send and receive the data effectively and precisely. And no loss of data can be assured as well.

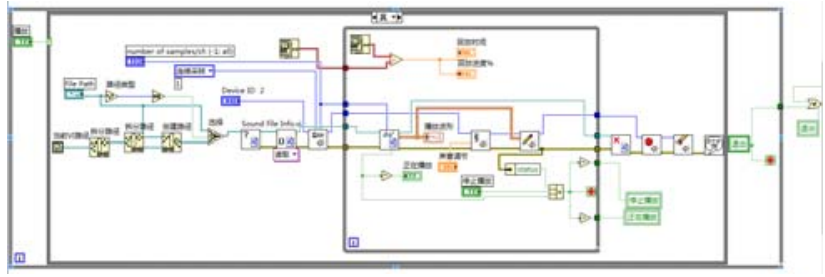

Figure7 The sound playback program

The sound playback program is showing as the figure7. The structure of this program is similar to the collection program. Click a switch to jump into the conditional loop. Then the system will process the path of the file which is going to be broadcasted then we can get the absolute path. Connect them to the "Sound File Info.vi" that we can get the data from the sound file. Subsequently, to configure the devices that can generate the sound. Before we want to display the sound waveform, it needs to call the "Sound File Read.vi”. We can get the sound data file as a format of the waveform array. Finally, it will write the data into the sound output devices. And when push the stop playback buttons, it will break out of the inner while loop, close the file, stop to play the sound and clear all the data.

\section{The system test}

The system test concludes three parts. First part is the collection of the sound data, the display of the waveform and the analysis of the spectrum. The second part is to send the sound data to the client and display the waveform. The Last part is to playback the sound data.

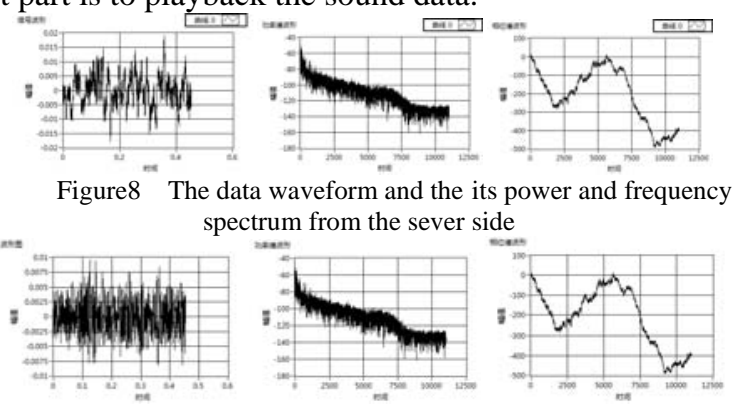


Figure9 The data waveform and the its power and frequency spectrum from the client side

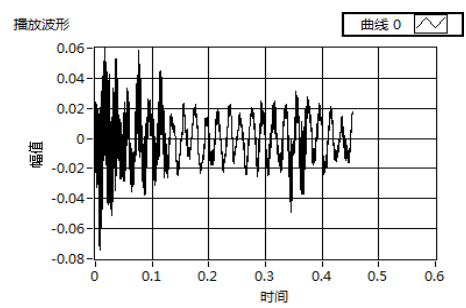

Figure10 The data playback waveform

The waveform of the sound data from the field and its power and frequency spectrum is showing as the figure8. What is corresponding to it is the as the figure9 shows, the waveform of the data from the client sides and its power and frequency spectrum. We can make a conclusion from the two figures that there is a slightly different between their waveforms. This is because the client sides delayed $100 \mathrm{~ms}$ than the server side. And the power and frequency spectrum we can consider that they are the same within the $100 \mathrm{~ms}$.

\section{CONCLUSION}

This paper is presented a system that approach to collect the sound data from the industry scene. It can process and analyze the data and transmit it to the client side timely. The aim to design it is that to help people monitor the sound signal in the high-risk environments conveniently. Practice shows us that the system has the advantages of operating simply and with friend human-computer interface. It can take the place of many complicated hardware devices that can save the costs to monitor. In addition, it can be further extended on this basis as needed.

\section{REFERENCE}

[1] Deichun Tong, BaoHeng Yao. The processing of Engineering Signal and Equipment diagnostic.[M].Beijing: Science Press,2008.

[2] Fuming Wang. The Program Design of LabVIEW and Virtual Instruments[M].Xi'an: Xidian University Press,2009.

[3] Rui Li, Bing Zhou. LabVIEW 2009 Virtual Instruments From Entry to Master[M].Beijing: China Machine PRESS,2010.

[4] QIzhen Ruan. The Ten-year Experience of programming From A LabVIEW engineer and I[M].Beijing: Beijing aerospace press,2010.

[5] Jiping Wang, Zhe zhao. The research on Communication and Measurement Technology Based on LabVIEW[J].Automation and Instrumentation,2011,(01).

[6] Jiqing Han, Lei Zhang.Speech Signal Processing. Beijing:Tsinghua University Press,2005

[7] Feng Pei, Wansheng, Yang. Hybrid Programming by LabVIEW and MATLAB[J]. Application of Electronic Technique,2004,(03). 